\title{
Linear multiscale analysis and finite element validation of stretching and bending dominated lattice materials
}

\author{
Andrea Vigliotti, Damiano Pasini* \\ McGill University, Mechanical Engineering Dept.
}

\begin{abstract}
The paper presents a multiscale procedure for the linear analysis of components made of lattice materials. The method allows the analysis of both pin-jointed and rigid jointed microstruss materials with arbitrary topology of the unit cell. At the macroscopic level, the procedure enables to determine the lattice stiffness, while at the microscopic level the internal forces in the lattice elements are expressed in terms of the macroscopic strain applied to the lattice component. A numeric validation of the method is described. The procedure is completely automated and can be easily used within an optimization framework to find the optimal geometric parameters of a given lattice material.
\end{abstract}

Keywords: Cellular Materials, Lattice Materials, Microtruss Materials, Multiscale Mechanics, Finite Element Modelling

\section{Introduction}

Cellular materials are a broad range of natural and artificial materials characterized by an abundance of microvoids confined in cells. The macroscopic characteristics of a cellular material depend not only on the shape and volume of the voids, but also on the material and cross section of the cell walls. As a subset of cellular materials, lattice materials are characterised by an ordered periodic microstructures obtained by replicating a unit cell

*corresponding author

Email addresses: andrea.vigliotti@mail.mcgill.ca (Andrea Vigliotti), damiano.pasini@mcgill.ca (Damiano Pasini) 
along independent tessellation vectors. For a given density, lattice materials are tenfold stiffer and threefold stronger than foams, which, due to their stochastic arrangement of cells, lie below the lattices in the Ashbys charts (Ashby, 2005).

Recent developments in additive manufacturing enable to build lattice materials with a high level of quality at affordable cost (Yang et al., 2002; Stampfl et al., 2004). Such techniques provide material designers with a superior degree of control on the material properties and allow them to tailor the material performance to meet prescribed multifunctional requirements. For instance, desired macroscopic stiffness, strength, and collapse mode can be attained in given directions by properly selecting the geometric parameters of the microstructure. Unusual mechanical behaviour, such as negative macroscopic Poisson's ratio, can be obtained by selecting auxetic topologies of the lattice (Lakes, 1987). In the aerospace sector, lattice materials can be applied for the design of morphing wings for next generation aircrafts (Spadoni, 2007; Alderson and Alderson, 2007; Gonella and Ruzzene, 2008). In the biomedical field, lattice materials have been proposed for advanced bone-replacement prosthesis, where the microtruss can be designed to resemble the inner architecture of trabecular bones, allowing seamless bone-implant integration, with reduced stress-shielding and bone resorption (Murr, 2010).

Reliable constitutive models are necessary to accurately predict the properties of lattice materials and exploit fully their potential. If the microscopic dimensions of the lattice are small compared to the macroscopic dimensions of the component, the number of degrees of freedom of a detailed model becomes extremely large and a direct approach involving the individual modelling of each cell is not practical.

An abundance of literature exists about constitutive models for cellular and lattice materials. In a work discussing alternative approaches for the analysis of large periodic structures, Noor (1988) emphasized that modelling the discrete structure as an equivalent continuum is the most promising strategy. He also outlined a method to evaluate the elastic constants of the surrogate continuum based on the isolation of the repeating cell and the use of the Taylor series expansion to approximate the displacement field inside the cell. His conclusion is that the Cauchy strain tensor can be used for the analysis of pin-jointed lattices, while for rigid jointed lattices the micropolar strain theory should be adopted.

In their comprehensive work on cellular materials, Gibson and Ashby (1988) estimated the stiffness and the strength of hexagonal and cubic lat- 
tices considering only bending in the cell walls. Their analysis focuses on a single cell under uni-axial load conditions and models the cell walls as either beams or plates. Zhu et al. (1997) applied a similar approach to model the tetrakaidecahedral topology, the cell shape usually assumed by foams, and obtained the Young's and shear moduli as a function of the relative density.

Wang et al. (2005) analysed the behaviour of extruded beams with cellular cross section, subjected to combined in-plane and out-of plane loadings. The in-plane macroscopic stiffness of the beam cross section was derived for a number of bidimensional lattices, considering a single cell subjected to shear and compression along different axes. The elastic constants of the lattices were determined through a detailed analysis of each case, the pertinent loads were applied to the unit cell, and the lattice stiffness was calculated from the resulting nodal displacements (Wang and McDowell, 2004). Kumar and McDowell (2004), on the other hand, used the micropolar theory to estimate the stiffness of rigid-jointed lattices. The rotational components of the micropolar field were used to account for nodal rotations. The displacements and rotations within the unit cell were expressed by a second order Taylor expansion about the cell centroid; then, the micropolar constitutive constants were determined by equating the expressions of the deformation energy of the micropolar continuum and of the discrete lattice. The analysis was limited to unit cell topologies that included a single internal node only. Lately, Gonella and Ruzzene (2008) analysed the wave propagation in repetitive lattices by considering an equivalent continuous media; the generalized displacements field of the unit cell was expressed by a Taylor series expansion around a reference node; the equivalent elastic properties were obtained by direct comparison of the wave equations of the homogenized model, and of a uniform plate under plane-stress. The method was illustrated with specific reference to the regular Hexagonal and re-entrant honeycombs. The same authors, in a more recent paper (Gonella and Ruzzene, 2010), noted that the order of the Taylor series expansion is limited by the number of boundary conditions that can be imposed on the unit cell, and limits the accuracy of the continuous model; the authors, thus, proposed an alternative approach using multiple cells as repeating units to improve the capability of the continuous model in capturing local deformation modes. Another approach was recently presented by Hutchinson and Fleck (2006), who resorted to the Bloch theorem and the Cauchy-Born rule to analyse pin-jointed lattices materials with nodes only on the boundary of the unit cell. Elsayed and Pasini (2010a) expanded this method introducing the dummy node rule, for the analysis of 
pin jointed lattices with elements intersecting the unit cell envelope. The same authors used this approach for the analysis of the compressive strength of columns made of lattice materials (Elsayed and Pasini, 2010b).

This paper presents an alternative method for the analysis of both pinjointed and rigid-jointed lattices. The procedure is based on a multiscale approach, where the macroscopic properties of the lattice are evaluated by expressing the microscopic deformation work as a function of the macroscopic strain field. In contrast to previous approaches relying on the Taylor series or the Cauchy-Born rule for the approximation of the displacements within the repeating cell, this method do not make any kinematic assumption on the internal points, but only on the boundary points of the cell. In addition, our approach does not resort to micropolar theory for the determination of the lattice nodal rotations; rather the rotational degrees of freedom (DoFs) of the cell nodes are evaluated enforcing periodic equilibrium conditions on the unit cell. At the microscopic level, after expressing the nodal DoFs of the unit cell as a function of the components of the macro-strain field, the internal forces in the lattice members are determined to verify whether the solid material of the cells fails. The procedure is illustrated with reference to three bidimensional topologies, namely the triangular, the hexagonal and the Kagome lattice. Its validation is accomplished by comparing the displacements of a finite lattice to those of an equivalent continuous model for prescribed geometry of the component, applied loads and boundary conditions.

\section{The multiscale approach}

Lattices consist of a regular network of structural elements connected at joints; they are obtained by the replica of a unit cell along independent periodic vectors. Figure 1 shows the sample lattices under investigation in this paper.

A multiscale structural problem can be solved setting two boundary value problems, one at the component level, and the other at the microscopic level; the solution can be found by defining proper relations between the micro and macroscale models. Figure 2 summarizes the steps followed in setting up the multiscale framework. The procedure is general and can be used to account also for non linear lattice behaviour, such as geometric non linearity, due to the re-orientation of the lattice elements during loading. We follow the approach outlined by Kouznetsova et al. (2002). At the macro- 


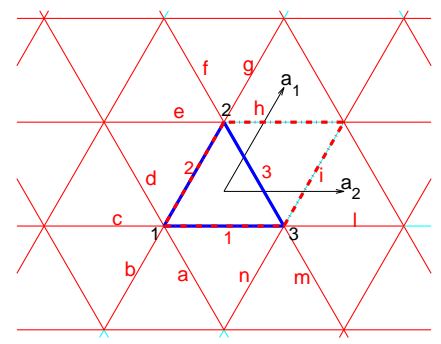

(a) Triangular lattice

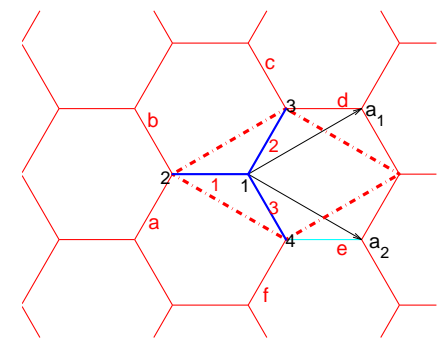

(b) Hexagonal lattice

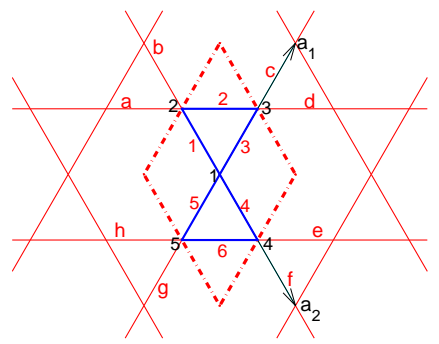

(c) Kagome lattice

Figure 1: Sample lattice topologies, $\mathbf{a}_{1}$ and $\mathbf{a}_{2}$ are periodic translational vectors, dotted lines represent cell boundaries and thicker lines the unit cell elements

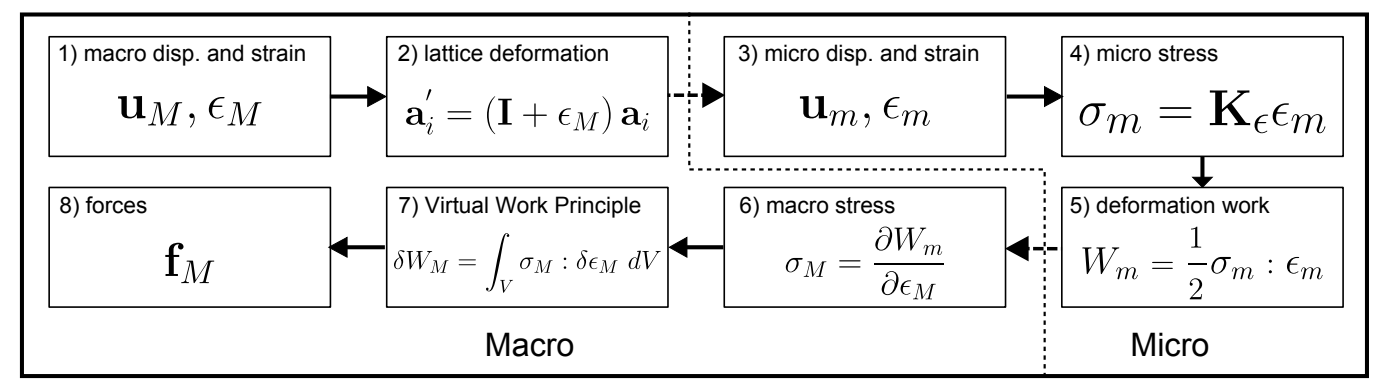

Figure 2: The multiscale scheme

scopic level (1), the components of the Cauchy strain tensor are evaluated from the displacements of the continuous medium, $\mathbf{u}_{M}$. We note here that although the macroscopic strain distorts the lattice, after deformation the microtruss remains periodic, and the deformed tessellation vectors comply with the macroscopic strain (2). At the microscale, the equilibrium problem of the unit cell (3-4-5) can be solved by imposing a kinematic and a static condition on the boundary of the cell. The relative displacements of the boundary points have to respect any change in the tessellation vectors, and the forces of the boundary nodes have to balance the forces imposed by the surrounding cells. Upon solving the equilibrium, the nodal DoFs, $\mathbf{u}_{m}$, and the deformation work, $\mathbf{W}_{m}$, of the unit cell can be determined. Since Cauchy stress and strain are work conjugate, the macroscopic stress tensor can be evaluated as shown in step (6). The equilibrium of the structure can then be solved by application of the Virtual Work Principle (7-8). We note that at the microscopic level, once the relation between macroscopic stress 
and nodal displacements of the unit cell is found, the internal forces in each element of the lattice can be obtained from the macro-strain components, as illustrated in section 4 .

\section{Lattice macroscopic stiffness}

The periodic nature of the lattices allows obtain the position of all the nodes of the infinite lattice, starting from the position of the nodes of the unit cell as follows

$$
\begin{array}{ll}
\mathbf{r}_{k}(\mathbf{l})=\mathbf{r}_{k}+l_{i} \mathbf{a}_{i} \quad & \forall l_{i} \in \mathcal{N} \\
& \text { and } k=1 \ldots J
\end{array}
$$

Where $\mathbf{r}_{k}$ is the position of the $k-t h$ node of the unit cell; $\mathbf{r}_{k}(\mathbf{l})$ are the positions of the nodes corresponding to $\mathbf{r}_{k} ; \mathbf{a}_{i}$ are the translational vectors; $i \in\{1,2\}$ for $2 D$ and $i \in\{1,2,3\}$ for $3 D$ lattices; $J$ is the number of nodes of the unit cell. As $l_{i}$ s span the integer field, $\mathcal{N}$, and $k$ spans the unit cell nodes, $r_{k}(l)$ spans of all the nodes of the lattice.

The unit cell nodes can be divided into two classes: i) the internal nodes, that only connect elements of the same cell, and have no correspondent node in the cell; and ii) the boundary nodes, that join elements of neighbour cells, and have necessarily at least one correspondent cell node on the opposite boundary. The position of each node of the lattice can then be obtained from a subset of the unit cell nodes, the independent nodes. We note that all internal nodes are independent, since no other node of the same cell can be obtained through a translation along any combination of the periodic vectors. For each cell topology, alternative choices of the boundary independent nodes are possible.

With reference to the triangular lattice (figure 1a), the position of nodes 2 and 3 can be obtained from the position of node 1 , as $\mathbf{r}_{2}=\mathbf{r}_{1}+\mathbf{a}_{1}$ and $\mathbf{r}_{3}=$ $\mathbf{r}_{1}+\mathbf{a}_{2}$, thus the triangular lattice has no internal nodes and one independent node. For the hexagonal lattice (figure $1 \mathrm{~b}$ ), it results $\mathbf{r}_{3}=\mathbf{r}_{2}+\mathbf{a}_{1}$ and $\mathbf{r}_{4}=\mathbf{r}_{2}+\mathbf{a}_{2}$, and the following independent nodes can then be defined: nodes 1, that is internal, and node 2. Analogously, for the Kagome lattice (figure 1c) a possible choice of independent nodes is nodes 1, 2, and 3; where node 1 is internal, and it results: $\mathbf{r}_{4}=\mathbf{r}_{2}-\mathbf{a}_{1}$ and $\mathbf{r}_{5}=\mathbf{r}_{3}-\mathbf{a}_{2}$.

Under the action of a uniform macroscopic strain field, the lattice will deform. The deformed periodic directions can be related to the components of 
the macroscopic strain tensor by means of the following (Asaro and Lubarda, 2006)

$$
\mathbf{a}_{i}^{\prime}=(\mathbf{I}+\boldsymbol{\epsilon}) \mathbf{a}_{i}
$$

where $\mathbf{I}$ is the unit tensor and $\boldsymbol{\epsilon}$ is the Cauchy strain tensor, whose components are

$$
\epsilon_{i j}=\frac{1}{2}\left(\frac{\partial u_{i}}{\partial x_{j}}+\frac{\partial u_{j}}{\partial x_{i}}\right)
$$

The position $\mathbf{r}_{k}^{\prime}$ of the $k-t h$ node of the deformed lattice will be given by the following

$$
\mathbf{r}_{k}^{\prime}(\mathbf{l})=\mathbf{r}_{k}^{\prime}+l_{i} \mathbf{a}_{i}^{\prime}=\mathbf{r}_{k}^{\prime}+l_{i}(\mathbf{I}+\boldsymbol{\epsilon}) \mathbf{a}_{i}
$$

Finally, the displacements of all the nodes of the lattice can be expressed as a function of the displacements of the independent nodes of the unit cell and of the components of the strain field as follows

$$
\mathbf{d}_{k}(\mathbf{l})=\mathbf{r}_{k}^{\prime}(\mathbf{l})-\mathbf{r}_{k}(\mathbf{l})=\mathbf{d}_{k}+l_{i} \boldsymbol{\epsilon} \mathbf{a}_{i}
$$

With reference to the unit cell, we introduce the array $\mathbf{d}$ containing the all the DoFs of of the unit cell nodes, and the array $\mathbf{d}_{0}$ containing only the DoFs of the independent nodes. Through equation (5), $\mathbf{d}$ can be expressed as a function of $\mathbf{d}_{0}$ and of the strain components, collected in the array $\mathbf{e}$, as

$$
\mathbf{d}=\mathbf{B}_{0} \mathbf{d}_{0}+\mathbf{B}_{e} \mathbf{e}
$$

where $\mathbf{B}_{0}$ is a block matrix of zero and unit matrices, and $\mathbf{B}_{e}$ is a block matrix mapping the relative displacements of the dependent nodes to the components of the macroscopic strain field. In the bidimensional case, the array of the components of the macroscopic strain field has three elements. Adopting the engineering notation for the components of the deformation field gives

$$
\mathbf{e}=\left[\epsilon_{x}, \epsilon_{y}, \gamma_{x y}\right]
$$

where $\gamma_{x y}=2 \epsilon_{x y}$.

With reference to the triangular lattice, since the only independent node is node 1 , the displacements of node 2 and 3 can be expressed as

$$
\begin{aligned}
& \mathbf{d}_{2}=\mathbf{d}_{1}+\boldsymbol{\epsilon} \mathbf{a}_{1} \\
& \mathbf{d}_{3}=\mathbf{d}_{1}+\boldsymbol{\epsilon} \mathbf{a}_{2}
\end{aligned}
$$


from which $\mathbf{d}, \mathbf{d}_{0}, \mathbf{B}_{0}$ and $\mathbf{B}_{e}$ are written as

$$
\mathbf{d}=\left[\begin{array}{l}
\mathbf{d}_{1} \\
\mathbf{d}_{2} \\
\mathbf{d}_{3}
\end{array}\right], \quad \mathbf{B}_{0}=\left[\begin{array}{l}
\mathbf{I} \\
\mathbf{I} \\
\mathbf{I}
\end{array}\right], \quad \mathbf{d}_{0}=\left[\begin{array}{l}
\mathbf{d}_{1}
\end{array}\right], \quad \mathbf{B}_{e}=\left[\begin{array}{c}
\mathbf{0} \\
\mathbf{B}_{e_{2}} \\
\mathbf{B}_{e_{3}}
\end{array}\right]
$$

where $\mathbf{I}$ is a diagonal matrix and the sub-matrices $\mathbf{B}_{e_{2}}$ and $\mathbf{B}_{e_{3}}$ map the DoF of the nodes 2 and 3 to the components of the strain field. Section 5 illustrates in detail how the elements of $\mathbf{B}_{e}$ are formed.

For the hexagonal lattice, following the distinction between dependent and independent nodes it results

$$
\begin{aligned}
& \mathbf{d}_{3}=\mathbf{d}_{2}+\boldsymbol{\epsilon} \mathbf{a}_{1} \\
& \mathbf{d}_{4}=\mathbf{d}_{2}+\boldsymbol{\epsilon} \mathbf{a}_{2}
\end{aligned}
$$

from which the following $\mathbf{B}_{0}$ and $\mathbf{B}_{e}$ matrices are obtained

$$
\mathbf{d}=\left[\begin{array}{l}
\mathbf{d}_{1} \\
\mathbf{d}_{2} \\
\mathbf{d}_{3} \\
\mathbf{d}_{4}
\end{array}\right], \quad \mathbf{B}_{0}=\left[\begin{array}{ll}
\mathbf{I} & 0 \\
0 & 1 \\
0 & 1 \\
0 & \mathbf{I}
\end{array}\right], \quad \mathbf{d}_{0}=\left[\begin{array}{l}
\mathbf{d}_{1} \\
\mathbf{d}_{2}
\end{array}\right], \quad \mathbf{B}_{e}=\left[\begin{array}{c}
0 \\
0 \\
\mathbf{B}_{e_{3}} \\
\mathbf{B}_{e_{4}}
\end{array}\right]
$$

For the Kagome lattice, we can write

$$
\begin{aligned}
& \mathbf{d}_{4}=\mathbf{d}_{2}+\boldsymbol{\epsilon} \mathbf{a}_{2} \\
& \mathbf{d}_{5}=\mathbf{d}_{3}-\boldsymbol{\epsilon} \mathbf{a}_{1}
\end{aligned}
$$

which yields the following

$$
\mathbf{d}=\left[\begin{array}{l}
\mathbf{d}_{1} \\
\mathbf{d}_{2} \\
\mathbf{d}_{3} \\
\mathbf{d}_{4} \\
\mathbf{d}_{5}
\end{array}\right], \quad \mathbf{B}_{0}=\left[\begin{array}{lll}
\mathbf{I} & 0 & 0 \\
0 & 1 & 0 \\
0 & 0 & \mathbf{I} \\
0 & 1 & 0 \\
0 & 0 & \mathbf{I}
\end{array}\right], \quad \mathbf{d}_{0}=\left[\begin{array}{l}
\mathbf{d}_{1} \\
\mathbf{d}_{2} \\
\mathbf{d}_{3}
\end{array}\right], \quad \mathbf{B}_{e}=\left[\begin{array}{c}
0 \\
0 \\
0 \\
\mathbf{B}_{e_{4}} \\
\mathbf{B}_{e_{5}}
\end{array}\right]
$$

The array $\mathbf{d}_{0}$ in equation (6) will be determined by imposing the selfequilibrium condition on the nodal forces of the unconstrained unit cell.

By means of the finite element method, the nodal forces of the unit cell, f, can be expressed in terms of nodal DoFs as

$$
\mathbf{f}=\mathbf{K}_{u c} \mathbf{d}
$$

where $\mathbf{K}_{u c}$ is the unit cell stiffness matrix, and $\mathbf{d}$, is the array of nodal DoFs. After deformation, all nodal forces must be zero in the infinite lattice because 
no body forces are applied to the lattice. This condition can be expressed in terms of the nodal forces of a single cell and can be written as

$$
\mathbf{A}_{0} \mathbf{f}=\mathbf{0}
$$

where $\mathbf{A}_{0}$, the equilibrium matrix, is a block matrix, whose entries are either unit or zero matrices, and depends on the unit cell topology and the periodic directions.

With reference to the triangular lattice, the equilibrium condition for the node 1 is that the sum of all the forces, due to the edges connecting in node 1 is zero. Because of the periodicity, the sum of the forces due to edges $c$ and $d$ is equal to the sum of the forces due to edges 1 and 3, and the sum of the forces due to edges $a$ and $b$ is equal to the sum of the forces due to edges 2 and 3. Therefore, a condition for the equilibrium of the node 1 can be expressed in terms of the nodal forces of the unconstrained unit cell as

$$
\mathbf{f}_{1}+\mathbf{f}_{2}+\mathbf{f}_{3}=\mathbf{0}
$$

Following a similar reasoning, two identical equations are obtained for the other nodes. Therefore, the matrix $\mathbf{A}_{\mathbf{0}}$ for the triangular lattice is the following

$$
\mathbf{A}_{0}=\left[\begin{array}{lll}
\mathbf{I} & \mathbf{I} & \mathbf{I}
\end{array}\right]
$$

With reference to the Hexagonal lattice, the equilibrium conditions are

$$
\begin{array}{ll}
(i) & \mathbf{f}_{1}=\mathbf{0} \\
(\text { ii }) & \mathbf{f}_{2}+\mathbf{f}_{3}+\mathbf{f}_{4}=\mathbf{0}
\end{array}
$$

the above can be justified as follows: (i) node 1 is internal and $\mathbf{f}_{1}$ is the sum of all element forces acting on it; equation (ii) follows to satisfy the periodicity condition, which requires that a) the force due to edge $a$ on node 2 be equal to the force due to edge 2 on node 3 , and $b$ ) the force due to edge $b$ on node 2 be equal to the force due to edge 3 on node 4 . The resulting equilibrium matrix is

$$
\mathbf{A}_{0}=\left[\begin{array}{llll}
\mathbf{I} & 0 & 0 & 0 \\
0 & \mathbf{I} & \mathbf{I} & \mathbf{I}
\end{array}\right]
$$

Similar reasoning applies to the Kagome lattice, for which the following relations hold

$$
\begin{array}{ll}
(i) & \mathbf{f}_{1}=\mathbf{0} \\
(\text { ii }) & \mathbf{f}_{2}+\mathbf{f}_{4}=\mathbf{0} \\
(\text { iii }) & \mathbf{f}_{3}+\mathbf{f}_{5}=\mathbf{0}
\end{array}
$$


where: $(i)$ node 1 is internal; $(i i)$ node 2 connects edge 1 and edge 2 , whose sum is $\mathbf{f}_{2}$, and edge $a$ and edge $b$, whose sum is $\mathbf{f}_{4} ;$ (iii) node 3 connects edge 2 and edge 3 , whose sum is $\mathbf{f}_{3}$, and edge $c$ and edge $d$, whose sum is $\mathbf{f}_{5}$. For the Kagome lattice the equilibrium matrix is

$$
\mathbf{A}_{0}=\left[\begin{array}{lllll}
\mathbf{I} & 0 & 0 & 0 & 0 \\
0 & \mathbf{I} & 0 & \mathbf{I} & 0 \\
0 & 0 & \mathbf{I} & 0 & \mathbf{I}
\end{array}\right]
$$

By examining the way in which these matrices are constructed, we can verify that $\mathbf{A}_{0}=\mathbf{B}_{0}^{T}$. Thus, combining equations (14) and (15), the equilibrium equation can be written as

$$
\mathbf{A}_{0} \mathbf{K}_{u c} \mathbf{d}=\mathbf{B}_{0}^{T} \mathbf{K}_{u c} \mathbf{d}=\mathbf{0}
$$

Using the expression of equation (6) for $\mathbf{d}$ we obtain the following

$$
\mathbf{B}_{0}^{T} \mathbf{K}_{u c}\left(\mathbf{B}_{0} \mathbf{d}_{0}+\mathbf{B}_{e} \mathbf{e}\right)=\mathbf{0}
$$

from which the displacements of the independent nodes can be found in terms of e. From (23), the following linear system of equations results in the unknown $\mathbf{d}_{0}$ :

$$
\mathbf{B}_{0}^{T} \mathbf{K}_{u c} \mathbf{B}_{0} \mathbf{d}_{0}=-\mathbf{B}_{0}^{T} \mathbf{K}_{u c} \mathbf{B}_{e} \mathbf{e}
$$

Since both the lhs (left-hand side) and rhs (right-hand side) of the above equation belong to the column range of $\mathbf{B}_{0}^{T} \mathbf{K}_{u c}$, a solution will always exist. Nevertheless, since $\mathbf{K}_{u c}$ is the stiffness matrix of the unconstrained unit cell, its null space is not empty, and the solution to (24) is not unique; rather, the solution is given by an affine subspace defined by any particular solution of (24) and the null space of the matrix (Strang, 1988). In Section 3.1, we show that all solutions produce identical expressions for the deformation work and the macroscopic stiffness matrix, which are then unique.

Furthermore, we observe that equation (24) represents the equilibrium of the unit cell constrained by the surrounding cells. Its rhs is the residual on the equilibrium equation (15), with sign changed, resulting from the macroscopic strain field, if the independent DoFs, $\mathbf{d}_{0}$, are kept fixed. The lhs of the equation represents the residual on the equilibrium equation when the independent DoFs are not null, and no strain is applied to the lattice. Thus, given an arbitrary macroscopic strain field, the solutions of equation (24) are the independent DoFs that guarantee the equilibrium of the cell with its surrounding. Finally, substituting $\mathbf{d}_{0}$ and $\mathbf{e}$ in equation (6), we obtain the 
DoFs of the unit cell nodes that both comply with the macroscopic strain field and guarantee the equilibrium of the unit cell with its surroundings.

The particular solution to equation (24) is given by the following

$$
\mathbf{d}_{0}=-\left(\mathbf{B}_{0}^{T} \mathbf{K}_{u c} \mathbf{B}_{0}\right)^{+} \mathbf{B}_{0}^{T} \mathbf{K}_{u c} \mathbf{B}_{e} \mathbf{e}=\mathbf{D}_{0} \mathbf{e}
$$

where $(\cdot)^{+}$is the Moore-Penrose pseudoinverse. The displacements of all nodes of the unit cell can be obtained by substituting equation (25) into (6), which results in

$$
\mathbf{d}=\left(\mathbf{B}_{0} \mathbf{D}_{0}+\mathbf{B}_{e}\right) \mathbf{e}=\mathbf{D}_{e} \mathbf{e}
$$

Since e has three components, $\mathbf{D}_{e}$ will have three columns. Each column represents the DoFs of the nodes of the unit cell, corresponding to the unit strain, for each strain component. Thus, the array $\mathbf{D}_{e}$ effectively links the components of the macroscopic strain to the DoFs of the unit cell nodes, for an arbitrary strain. Furthermore, $\mathbf{D}_{e}$ allows express the specific lattice deformation work as a function of the macroscopic strain components as

$$
W=\frac{1}{2 S_{\mathrm{uc}}} \mathbf{e}^{T} \mathbf{D}_{e}^{T} \mathbf{K}_{u c} \mathbf{D}_{e} \mathbf{e}
$$

where $S_{\mathrm{uc}}$ is the area of the unit cell. Since the linearised Cauchy stress and strain are work conjugate Slaughter (2002), it results $\sigma_{i j}=\frac{\partial W}{\partial \epsilon_{j i}}$, that makes possible to express the components of lattice macroscopic stiffness tensor as $E_{i j h k}=\frac{\partial \sigma_{i j}}{\partial \epsilon_{h k}}$, resulting in

$$
\mathbf{K}=\frac{1}{S_{\mathrm{uc}}} \mathbf{D}_{e}^{T} \mathbf{K}_{u c} \mathbf{D}_{e}
$$

We note that three matrices are required to evaluate matrix $\mathbf{D}_{e}$, specifically: (i) the stiffness matrix of the unconstrained unit cell, $\mathbf{K}_{u c}$, easily obtained by means of standard finite element procedure; (ii) the block matrix $\mathbf{B}_{0}^{T}$ defines the periodic boundary conditions, and it depends on the periodic translational vectors and on the cell topology; similarly (iii) $\mathbf{B}_{\epsilon}$ expresses the relative displacements of the boundary nodes as a function of the components of the macroscopic strain field. Once these matrices have been assembled, the macroscopic stiffness of the lattice can be calculated by means of equations (25) and (26). Thus the evaluation of the lattice stiffness for alternative geometric properties of the cell edges can be completely automated, and integrated within an optimization framework (Vigliotti and Pasini, 2011). 


\subsection{Uniqueness of $W$ and $\mathbf{K}$}

As described earlier, the system of equations (24) has always a solution, although not unique. In this section, we show that all solutions of (24) produce the same mechanical work and, consequently, the same macroscopic stiffness matrix.

Since the null space of the matrix $\mathbf{B}_{0}^{T} \mathbf{K}_{u c} \mathbf{B}_{0}$ is not empty, the general solution to equation (24) is an affine subspace given by

$$
\mathbf{d}_{0}=\mathbf{D}_{0} \mathbf{K}+\boldsymbol{\mu} \quad \forall \boldsymbol{\mu} \in \operatorname{Null}\left(\mathbf{B}_{0}^{T} \mathbf{K}_{u c} \mathbf{B}_{0}\right)
$$

where $\mathbf{D}_{0} \mathbf{K}$ is a particular solution, and $\boldsymbol{\mu}$ is any element of $\operatorname{Null}\left(\mathbf{B}_{0}^{T} \mathbf{K}_{u c} \mathbf{B}_{0}\right)$. Substituting the above in (26) it gives

$$
\mathbf{d}=\mathbf{B}_{0}\left(\mathbf{D}_{0} \mathbf{e}+\boldsymbol{\mu}\right)+\mathbf{B}_{e} \mathbf{e}=\mathbf{D}_{e} \mathbf{e}+\mathbf{B}_{0} \boldsymbol{\mu}
$$

thus the expression for the deformation work is

$$
\begin{aligned}
W & =\frac{1}{2 S_{\mathrm{uc}}}\left(\boldsymbol{\mu}^{T} \mathbf{B}_{0}^{T}+\mathbf{e}^{T} \mathbf{D}_{e}^{T}\right) \mathbf{K}_{u c}\left(\mathbf{D}_{e} \mathbf{e}+\mathbf{B}_{0} \boldsymbol{\mu}\right) \\
& =\frac{1}{2 S_{\mathrm{uc}}}\left(\mathbf{e}^{T} \mathbf{D}_{e}^{T} \mathbf{K}_{u c} \mathbf{D}_{e} \mathbf{e}+\boldsymbol{\mu}^{T} \mathbf{B}_{0}^{T} \mathbf{K}_{u c} \mathbf{B}_{0} \boldsymbol{\mu}+2 \mathbf{e}^{T} \mathbf{D}_{e}^{T} \mathbf{K}_{u c} \mathbf{B}_{0} \boldsymbol{\mu}\right)
\end{aligned}
$$

Since the following holds

$$
\begin{aligned}
& \boldsymbol{\mu}^{T} \mathbf{B}_{0}^{T} \mathbf{K}_{u c} \mathbf{B}_{0} \boldsymbol{\mu}=0 \\
& \mathbf{K}_{u c}=\mathbf{K}_{u c}^{T}
\end{aligned} \quad \Longrightarrow \quad \mathbf{K}_{u c} \mathbf{B}_{0} \boldsymbol{\mu}=\mathbf{0}
$$

it follows that the last two terms in the parenthesis on the lhs of equation (31) are both zero. This proves that the expression of the deformation work does not depend on $\boldsymbol{\mu}$; therefore any solution of equation (24) will produce the same expression for the deformation work and the macroscopic stiffness matrix of the lattice.

\section{Lattice internal forces}

By means of the macroscopic stiffness obtained through equation (28), we can model lattice materials as uniform materials. After solving the structural equilibrium for a given component, the stress and strain of the equivalent uniform medium can be calculated. Being homogenised estimates, these figures do not represent the load on the solid material of the lattice. As a 
result, they cannot be used to assess the material resistance. To evaluate the load on the solid material, first, the nodal DoFs of the lattice have to be calculated by means of equation (26) as a function of the macroscopic strain. Then to assess the resistance at the microscopic level, the stress and strain in the cell elements can be determined by means of the unit cell model, and compared with the strength of the solid material.

For instance, if the edges of the lattice are modelled as Euler-Bernoulli beams, the edge stretching, $s$, and curvature, $\chi$, are given by (Zienkiewicz and Taylor, 2005)

$$
\begin{aligned}
& s=\frac{u_{2}-u_{1}}{L} \\
& \chi=x\left(6 \frac{\theta_{1}+\theta_{2}}{L^{2}}-12 \frac{v_{2}-v_{1}}{L^{3}}\right)-\left(2 \frac{2 \theta_{1}+\theta_{2}}{L}+6 \frac{v_{1}-v_{2}}{L^{2}}\right)
\end{aligned}
$$

where $x$ is the abscissa along the element, varying from 0 to $L$, the element length; $u_{i}$ are the axial components of the nodal displacements; $v_{i}$ are the transverse components; $\theta_{i}$ are the nodal rotations (figure 3 ). The normal force and the bending moment are, thus, given by

$$
\begin{aligned}
& N=E_{s} A s \\
& M=E_{s} I_{z z} \chi
\end{aligned}
$$

where $E_{s}$ is the Young modulus of the solid material of the lattice, $A$ and $I_{z z}$ are the cross section area and the second moment of inertia, respectively.

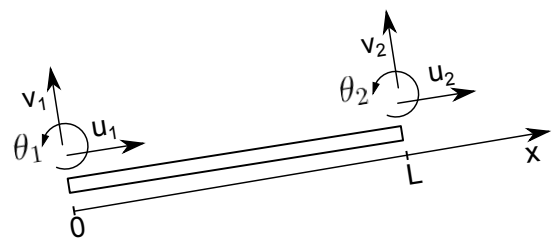

Figure 3: Degrees of Freedom of a lattice element

\section{Analysis of selected lattice topologies and model validation}

The procedure described in the previous section is here applied with reference to the lattice topologies reported in figure 1.

According to Gibson et al. (1982), for low density materials, cell walls behave as slender beams, and can be modelled as Euler-Bernoulli beams, neglecting shear. The stiffness matrix of the unit cell, $\mathbf{K}_{u c}$, can then be 
obtained by assembling the stiffness matrices of the single elements. Since this paper is restricted to a linear analysis, we can separate the stiffness matrix into the bending and stretching contributions, and obtain the following expressions of the strain energy due to bending and stretching:

$$
\begin{aligned}
& W_{b}=\frac{1}{2} \mathbf{d}^{T} \mathbf{K}_{u c_{b}} \mathbf{d} \\
& W_{s}=\frac{1}{2} \mathbf{d}^{T} \mathbf{K}_{u c_{s}} \mathbf{d}
\end{aligned}
$$

where $K_{u c_{s}}$ is the stiffness matrix for the axial deformation, and $K_{u c_{b}}$ is the stiffness matrix for the bending. The total deformation work of the unit cell is given by the $W=W_{s}+W_{b}$.

We note that the method presented in this paper is general. Although here the lattice edges have been modelled as Bernoulli beams, the cell walls can also be modelled with other type of elements, such as Timoshenko beams or plane membranes, as required by the structural function.

\subsection{Numerical validation}

To validate and assess the accuracy of the procedure described in the previous section, a finite rectangular plate made of lattice material is examined under prescribed loads and constraints. In one case, the lattice of the plate is modelled edge by edge using beam elements. In the other case, the rectangular plate is modelled with a uniform material of equivalent stiffness. The displacements of the free sides of the two models are then compared for each of the lattice topologies considered in this paper. The boundary conditions and the two load cases (figure 4) have been specified as follows

- On the constrained side, the nodes are pinned on both the continuous and the discrete model;

- On the side where the load is applied, the nodes are constrained to remain aligned, and the load is applied on one node only;

- The remaining sides are free.

Since the lattice stiffness is evaluated for a uniform macroscopic strain applied to the infinite lattice, the displacements of the detailed model will deviate from those of the equivalent model in the areas where the boundary conditions are applied, and the strain gradients are stronger. As shown by Phani and Fleck (2008), a lattice with finite dimensions develops a transition zone in the proximity of the boundaries, the boundary layer, where the lattice deformations are not uniform. In these regions, the wavelength of 

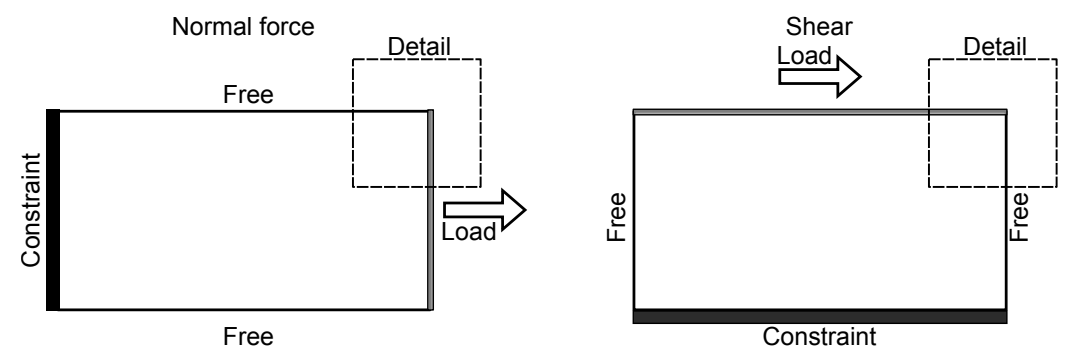

Figure 4: Boundary conditions and applied loads of the rectangular plate ("Detail" in Figures 6,8 and 10)

the macroscopic strain field becomes comparable to the length of the lattice edges; thus, in these zones the quality of the approximation of the continuous model tends to deteriorate.

The displacements of the continuous and discrete models are compared both quantitatively and qualitatively. The discrete model is chosen as a baseline, and the percentage differences between the two models have been evaluated interpolating the displacements of the continuous model at the position of the nodes of the discrete model. For each lattice topology, the maximum errors are given in a Table at the end of each section. For a qualitative assessment of the deformed shapes, the plots of the areas enclosed in the small rectangles at the top-left corner of the domains shown in figure 4, have been superimposed. We investigate the effect the length of the cells edges on the accuracy of the estimated stiffness. For this purpose, two lattices of different cell size have been considered for given outer dimensions of the rectangle. In each case, the lattice edges have been modelled with a single beam. The lattice with smaller cell size included a higher number of cells and of DoFs. The comparison between the models shows that the resulting estimate of the stiffness increases if the size of the cell decreases with respect to the size component. As expected, the accuracy of the results thus improves when the lattice is relatively closer to the asymptotic approximation of an infinite media.

The commercial software Ansys rev 12.1 (ANSYS, Inc.) is used for the numerical simulations. In particular, for the detailed model, the BEAM3 element, which models a bi-dimensional Bernoulli beam, has been used for the cell elements, whose material properties and geometry parameters are reported in Table 1. For the continuous model, plane-stress PLANE182 elements have been used with unit thickness. The PLANE182 element has 
only translational degrees of freedom and allows the input of an arbitrary material stiffness matrix.

\begin{tabular}{|l|c|l|c|}
\hline \multicolumn{2}{|c|}{ Cross-section } & \multicolumn{2}{|c|}{ Material } \\
\hline $\mathrm{A}$ & $\pi 0.1^{2}$ & $E_{s}$ & $110^{2}$ \\
\hline$I_{z z}$ & $\pi \frac{0.1^{4}}{4}$ & $\nu$ & 0.3 \\
\hline
\end{tabular}

Table 1: Cross-section and material properties of each truss element

\subsubsection{Triangular Lattice}

The unit cell and the translational vectors for the triangular lattice are illustrated in figure 1a. The translational vectors are $\mathbf{a}_{1}=L\left[\frac{1}{2}, \frac{\sqrt{3}}{2}\right]$, and $\mathbf{a}_{2}=L[1,0]$, the area of the unit cell is $S_{\mathrm{uc}}=\left|\mathbf{a}_{1} \times \mathbf{a}_{2}\right|=L^{2} \frac{\sqrt{3}}{2}$.

As mentioned earlier, the displacements of the dependent nodes can be expressed as a function of the displacements of the independent nodes and of the components of the deformation field. Equation (8) can be written in terms of the DoF of node 2 and 3 as

$$
\begin{array}{rlrl}
d_{2_{x}} & =d_{1_{x}}+\epsilon_{x} a_{1 x}+\frac{1}{2} \gamma_{x y} a_{1 y} & & d_{3_{x}}=d_{1_{x}}+\epsilon_{x} a_{2 x}+\frac{1}{2} \gamma_{x y} a_{2 y} \\
d_{2_{y}}=d_{1_{y}}+\frac{1}{2} \gamma_{x y} a_{1 x}+\epsilon_{y} a_{1 y} & & d_{3_{y}}=d_{1_{y}}+\frac{1}{2} \gamma_{x y} a_{2 x}+\epsilon_{y} a_{2 y} \\
d_{2_{\theta}}=d_{1_{\theta}} & d_{3_{\theta}}=d_{1_{\theta}}
\end{array}
$$

The elements of the sub-matrices $\mathbf{B}_{e_{1}}$ and $\mathbf{B}_{e_{2}}$ of equation (9) can thus be expressed by writing equations (36) in matrix form, as

$$
\begin{aligned}
& \mathbf{B}_{e_{1}}=\left[\begin{array}{ccc}
a_{1 x} & 0 & \frac{a_{1 y}}{2} \\
0 & a_{1 y} & \frac{a_{1 x}}{2} \\
0 & 0 & 0
\end{array}\right]=L\left[\begin{array}{ccc}
\frac{1}{2} & 0 & \frac{\sqrt{3}}{4} \\
0 & \frac{\sqrt{3}}{2} & \frac{1}{4} \\
0 & 0 & 0
\end{array}\right] \\
& \mathbf{B}_{e_{2}}=\left[\begin{array}{ccc}
a_{2 x} & 0 & \frac{a_{2 y}}{2} \\
0 & a_{2_{y}} & \frac{a_{2 x}}{2} \\
0 & 0 & 0
\end{array}\right]=L\left[\begin{array}{lll}
1 & 0 & 0 \\
0 & 0 & \frac{1}{2} \\
0 & 0 & 0
\end{array}\right]
\end{aligned}
$$

After evaluating the matrix $\mathbf{B}_{0}$, as described in section 3 and $\mathbf{B}_{e}$, as described above, it is possible to evaluate the matrix $\mathbf{D}_{e}$ by means of equations (25) and (26). 
All calculations can be performed symbolically by means of dedicated software packages. The expression of $\mathbf{D}_{e}$ is reported below

$$
\mathbf{D}_{e}^{T}=L\left[\begin{array}{ccccccccc}
0 & 0 & 0 & \frac{1}{2} & 0 & 0 & 1 & 0 & 0 \\
0 & 0 & 0 & 0 & \frac{\sqrt{3}}{2} & 0 & 0 & 0 & 0 \\
0 & 0 & 0 & \frac{\sqrt{3}}{4} & \frac{1}{4} & 0 & 0 & \frac{1}{2} & 0
\end{array}\right]
$$

As mentioned earlier, the columns of $\mathbf{D}_{e}$ represent the value of the nodal displacements and rotations corresponding to unit strains; they can be used to plot the deformed lattices, corresponding to each unitary strain state, as shown in figure 5 .

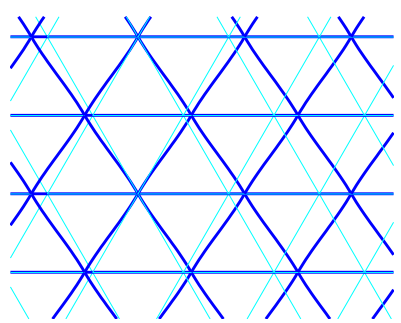

(a) $\epsilon_{x}>0, \epsilon_{y}=\gamma_{x y}=0$

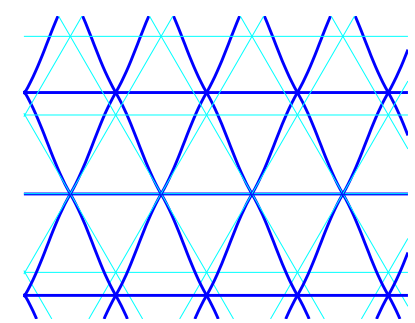

(b) $\epsilon_{y}>0, \epsilon_{x}=\gamma_{x y}=0$

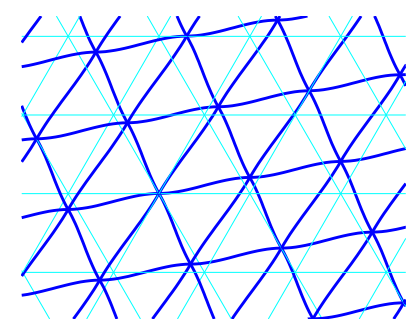

(c) $\gamma_{x y}>0, \epsilon_{x}=\epsilon_{y}=0$

Figure 5: Deformation modes of the triangular lattice with rigid joints

Through equation (27) and equation (28), we obtain the expressions for the deformation work and for the macroscopic stiffness matrix as

$$
\begin{aligned}
& W=\frac{\sqrt{3} E_{s}}{4}\left[\left(\frac{A}{L}\left(\gamma_{x y}{ }^{2}+3 \epsilon_{x}{ }^{2}+3 \epsilon_{y}{ }^{2}+2 \epsilon_{x} \epsilon_{y}\right)+\right.\right. \\
& \left.\frac{12 I_{z z}}{L^{3}}\left(\gamma_{x y}{ }^{2}+\left(\epsilon_{x}-\epsilon_{y}\right)^{2}\right)\right] \\
& \mathbf{K}=\frac{3 E_{s}}{4 L^{3} \sqrt{3}}\left[\begin{array}{ccc}
3\left(A L^{2}+4 I_{z z}\right) & A L^{2}-12 I_{z z} & 0 \\
A L^{2}-12 I_{z z} & 3\left(A L^{2}+4 I_{z z}\right) & 0 \\
0 & 0 & A L^{2}+12 I_{z z}
\end{array}\right]
\end{aligned}
$$

The stretching and bending contributions to strain energy are given by the following

$$
\begin{aligned}
& W_{s}=\frac{\sqrt{3} E_{s} A}{4 L}\left(\gamma_{x y}{ }^{2}+3 \epsilon_{x}^{2}+3 \epsilon_{y}^{2}+2 \epsilon_{x} \epsilon_{y}\right) \\
& W_{b}=\frac{3 \sqrt{3} E_{s} I_{z z}}{L^{3}}\left(\gamma_{x y}{ }^{2}+\left(\epsilon_{x}-\epsilon_{y}\right)^{2}\right)
\end{aligned}
$$

The triangular lattice is isotropic, and the eigenvectors of its macroscopic stiffness matrix are: $\boldsymbol{\epsilon}_{1}=[1,1,0], \boldsymbol{\epsilon}_{1}=[1,-1,0], \boldsymbol{\epsilon}_{1}=[0,0,1]$. Table 2 reports the eigenvalues of the stiffness matrix and the ratios $\frac{W_{b}}{W_{s}}$ corresponding 


\begin{tabular}{|l|c|c|c|}
\hline & $\boldsymbol{\epsilon}_{1}$ & $\boldsymbol{\epsilon}_{2}$ & $\boldsymbol{\epsilon}_{3}$ \\
\hline$\lambda$ & $\frac{2 \sqrt{3} E_{s} A}{L}$ & $\frac{\sqrt{3} E\left(A L^{2}+12 I_{z z}\right)}{L^{3}}$ & $\frac{\sqrt{3} E\left(A L^{2}+12 I_{z z}\right)}{4 L^{3}}$ \\
\hline$\frac{W_{b}}{W_{s}}$ & 0 & $\frac{12 I_{z z}}{A L^{2}}$ & $\frac{12 I_{z z}}{A L^{2}}$ \\
\hline
\end{tabular}

Table 2: Eigenvalues of the stiffness matrix and strain energy ratios for triangular lattice

to each eigenvector.

As shown in Table 2, for the hydrostatic stress, $\boldsymbol{\epsilon}_{1}$, no bending energy is present and the lattice is only subject to stretching. In the other cases, since is $o\left[I_{z z}\right]=o\left[A^{2}\right]$ and for slender beams is $o[A] \ll o\left[L^{2}\right]$, it results

$$
\frac{W_{b}}{W_{s}}=\frac{12 I_{z z}}{A L^{2}} \approx \frac{A}{L^{2}} \ll 1
$$

Thus the bending is always negligible with respect to stretching.

Once the nodal displacements have been found, the load in the lattice cell walls are determined through equation (34). Table 3 lists the normal forces and bending moments acting on each element for arbitrary strain components, at any point of the element.

\begin{tabular}{|c|c|}
\hline edge & Internal force \\
\hline 1 & $\begin{array}{l}N=E_{s} A \epsilon_{x} \\
M=E_{s} I_{z z}\left[\left(\frac{3}{L}-\frac{6}{L^{2}} x\right) \gamma_{x y}\right]\end{array}$ \\
\hline 2 & $\begin{array}{l}N=E_{s} A\left[\frac{1}{4} \epsilon_{x}+\frac{3}{4} \epsilon_{y}-\frac{\sqrt{3}}{4} \gamma_{x y}\right] \\
M=E_{s} I_{z z}\left[\left(\frac{3 \sqrt{3}}{2 L}-\frac{3 \sqrt{3}}{L^{2}} x\right) \epsilon_{x}+\left(\frac{3 \sqrt{3}}{L^{2}} x-\frac{3 \sqrt{3}}{2 L}\right) \epsilon_{y}+\left(\frac{3}{L^{2}} x-\frac{3}{2 L}\right) \gamma_{x y}\right.\end{array}$ \\
\hline 3 & $\begin{array}{l}N=E_{s} A\left[\frac{1}{4} \epsilon_{x}+\frac{3}{4} \epsilon_{y}+\frac{\sqrt{3}}{4} \gamma_{x y}\right] \\
M=E_{s} I_{z z}\left[\left(\frac{3 \sqrt{3}}{L^{2}} x-\frac{3 \sqrt{3}}{2 L}\right) \epsilon_{x}+\left(\frac{3 \sqrt{3}}{2 L}-\frac{3 \sqrt{3}}{L^{2}} x\right) \epsilon_{y}+\left(\frac{3}{L^{2}} x-\frac{3}{2 L}\right) \gamma_{x y}\right.\end{array}$ \\
\hline
\end{tabular}

Table 3: Normal forces and bending moments of the triangular lattice

The expressions in Table 3 allow verify if material failure occurs at the microscopic level. Once the structural problem is solved by using the homogenised representation of the material, and the components of the macro- 
scopic strain field are retrieved, the expressions in table 3 allow evaluate the load on each cell element and compare it with the beam strength.

Table 4 shows the maximum errors on the horizontal, $u$, and vertical, $v$, displacements of the two models; the deformed shape for the plate and the smaller beam model are illustrated in figure 6 .

\begin{tabular}{|c|c|c|c|c|c|}
\hline \multicolumn{2}{|c|}{ model DoF } & \multicolumn{2}{c|}{ Normal Load err. } & \multicolumn{2}{c|}{ Shear err. } \\
\hline beam & plate & $u$ err & $v$ err & $u$ err & $v$ err \\
\hline 5967 & 800 & $0.1 \%$ & $1.12 \%$ & $0.75 \%$ & $2.14 \%$ \\
\hline 58905 & 800 & $0.02 \%$ & $0.79 \%$ & $0.60 \%$ & $1.63 \%$ \\
\hline
\end{tabular}

Table 4: Errors of plate models wrt lattice models, triangular lattice

As can be observed, the maximum error is $2.14 \%$; furthermore the accuracy delivered by the plate model improves as the size of the lattice edges decreases with respect to the size of the component.

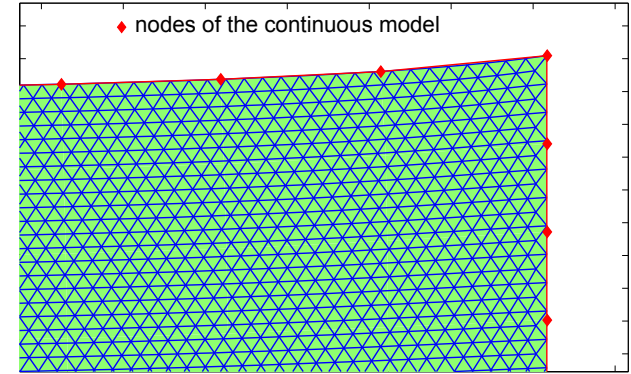

(a) Normal load, lattice and plate models

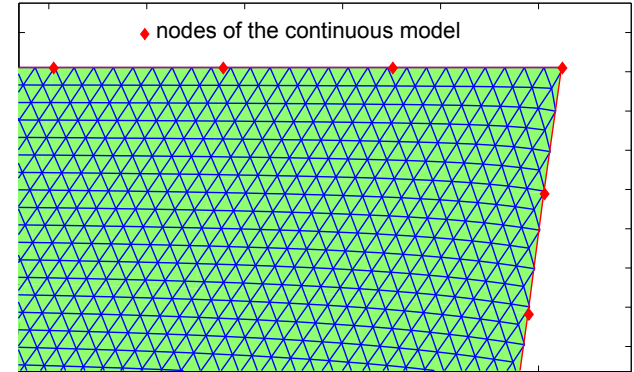

(b) Shear load, lattice and plate models

Figure 6: Deformed plate ("Detail" in Figure 4) made of triangular lattice with rigid joints

\subsubsection{Hexagonal Lattice}

The unit cell, and the translational vectors of the Hexagonal lattice are shown in figure $1 \mathrm{~b}$. The periodic directions are $\mathbf{a}_{1}=L\left[\frac{3}{2}, \frac{\sqrt{3}}{2}\right]$ and $\mathbf{a}_{2}=$ $L\left[\frac{3}{2},-\frac{\sqrt{3}}{2}\right]$; while the area of the unit cell is $S_{\mathrm{uc}}=\frac{3 \sqrt{3}}{2} L^{2}$. The $\mathbf{B}_{0}$ and $\mathbf{B}_{e}$ matrices for the hexagonal lattice are given by equation (11). The sub- 
matrices $\mathbf{B}_{e_{3}}$ and $\mathbf{B}_{e_{4}}$ are

$$
\begin{aligned}
& \mathbf{B}_{e_{3}}=\left[\begin{array}{ccc}
a_{1 x} & 0 & \frac{a_{1 y}}{2} \\
0 & a_{1_{y}} & \frac{a_{1 x}}{2} \\
0 & 0 & 0
\end{array}\right]=L\left[\begin{array}{ccc}
\frac{1}{2} & 0 & \frac{\sqrt{3}}{4} \\
0 & \frac{\sqrt{3}}{2} & \frac{1}{4} \\
0 & 0 & 0
\end{array}\right] \\
& \mathbf{B}_{e_{4}}=\left[\begin{array}{ccc}
a_{2 x} & 0 & \frac{a_{2 y}}{2} \\
0 & a_{2 y} & \frac{a_{2 x}}{2} \\
0 & 0 & 0
\end{array}\right]=L\left[\begin{array}{ccc}
\frac{1}{2} & 0 & \frac{-\sqrt{3}}{4} \\
0 & \frac{-\sqrt{3}}{2} & \frac{1}{4} \\
0 & 0 & 0
\end{array}\right]
\end{aligned}
$$

by means of equation (25) it is then possible to evaluate $\mathbf{D}_{e}$ for the hexagonal lattice

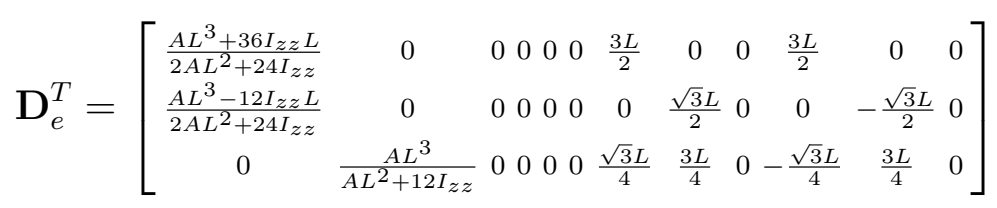

The deformed lattices corresponding to pure strains are shown in figure 7 . The expression of the deformation work and the macroscopic stiffness matrix are

$$
\begin{gathered}
W=\frac{E_{s} A}{4 \sqrt{3}\left(A L^{3}+12 I_{z z} L\right)}\left[A L^{2}\left(\epsilon_{x}+\epsilon_{y}\right)^{2}+\right. \\
\left.I_{z z}\left(2 \gamma_{x y}{ }^{2}+3 \epsilon_{x}{ }^{2}+3 \epsilon_{y}{ }^{2}-2 \epsilon_{x} \epsilon_{y}\right)\right] \\
\mathbf{K}=\frac{1}{2 \sqrt{3}} \frac{E_{s} A}{L\left(A L^{2}+12 I_{z z}\right)}\left[\begin{array}{ccc}
A L^{2}+36 I_{z z} & A L^{2}-12 I_{z z} & 0 \\
A L^{2}-12 I_{z z} & A L^{2}+36 I_{z z} & 0 \\
0 & 0 & 24 I_{z z}
\end{array}\right]
\end{gathered}
$$

The above expression for the macroscopic stiffness of the hexagonal lattice coincides with the findings of Gonella and Ruzzene (2008). Following the steps described in section 5, and using equation (35) the expressions for the bending and the stretching strain energy for the hexagonal lattice are found as a function of the strain field components, as

$$
\begin{aligned}
& W_{b}=\frac{4 \sqrt{3} A^{2} E s I_{z z} L\left(\epsilon_{x}{ }^{2}-2 \epsilon_{y} \epsilon_{x}+\gamma_{x y}{ }^{2}+\epsilon_{y}{ }^{2}\right)}{\left(A L^{2}+12 I_{z z}\right)^{2}} \\
& W_{s}=\frac{A E\left(A^{2}\left(\epsilon_{x}+\epsilon_{y}\right)^{2} L^{4}+24 A I_{z z}\left(\epsilon_{x}+\epsilon_{y}\right)^{2} L^{2}+144 I_{z z}^{2}\left(3 \epsilon_{x}{ }^{2}-2 \epsilon_{y} \epsilon_{x}+2 \gamma_{x y}{ }^{2}+3 \epsilon_{y}{ }^{2}\right)\right)}{2 \sqrt{3} L\left(A L^{2}+12 I_{z z}\right)^{2}}
\end{aligned}
$$

The hexagonal lattice is also isotropic, and the eigenvectors of its stiffness matrix are identical to those of the triangular lattice. Table 5 shows the eigenvalues of the stiffness matrix and the ratios $\frac{W_{b}}{W_{s}}$ corresponding to each 


\begin{tabular}{|l|c|c|c|}
\hline & $\boldsymbol{\epsilon}_{1}$ & $\boldsymbol{\epsilon}_{2}$ & $\boldsymbol{\epsilon}_{3}$ \\
\hline$\lambda$ & $\frac{2 E A}{\sqrt{3} L}$ & $\frac{16 \sqrt{3} E_{s} A I_{z z}}{A L^{3}+12 I_{z z} L}$ & $\frac{4 \sqrt{3} E_{s} A I_{z z}}{A L^{3}+12 I_{z z} L}$ \\
\hline$\frac{W_{b}}{W_{s}}$ & 0 & $\frac{A L^{2}}{12 I_{z z}}$ & $\frac{A L^{2}}{12 I_{z z}}$ \\
\hline
\end{tabular}

Table 5: Eigenvalues of the stiffness matrix and strain energy ratios of the hexagonal lattice with rigid joints

eigenvector. For hydrostatic stress, since contribution to lattice stiffness due to edges bending is again null, the strain energy is stored in stretching only. In the other cases, it can be noted that the deformation energy is mainly stored as bending, being $\frac{W_{b}}{W_{s}}=\frac{A L^{2}}{12 I_{z z}} \gg 1$, as expected since the lattice is bending dominated. We note that the eigenvalue corresponding to the hydrostatic stress is much higher than the others. With reference to $\frac{\lambda_{2}}{\lambda_{1}}$, it results

$$
\frac{\lambda_{2}}{\lambda_{1}}=\frac{24 I_{z z}}{A L^{2}+12 I_{z z}} \ll 1
$$

Thus, even if the deviatoric or shear stress components are small, the deformation in those directions will be dominant, and the lattice with fail according to the modes $\epsilon_{2}$ or $\epsilon_{3}$. Table 6 lists the normal forces and bending

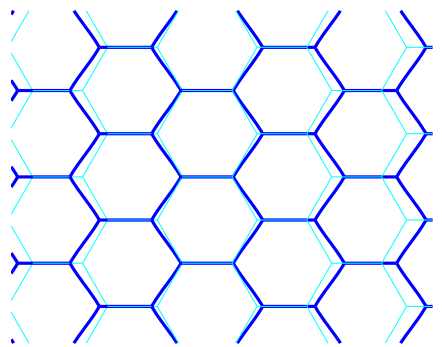

(a) $\epsilon_{x}>0, \epsilon_{y}=\gamma_{x y}=0$

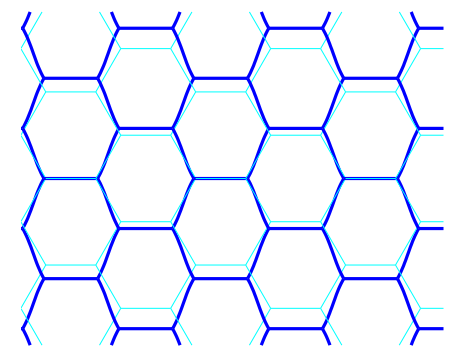

(b) $\epsilon_{y}>0, \epsilon_{x}=\gamma_{x y}=0$

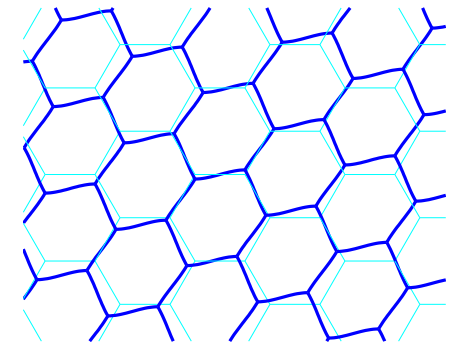

(c) $\gamma_{x y}>0, \epsilon_{x}=\epsilon_{y}=0$

Figure 7: Deformation modes of the hexagonal lattice with rigid joints

moments on each lattice element; Table 7 reports the displacement error of the plate model with respect to the lattice model; and Figure 8 illustrates the deformed shapes.

\subsubsection{Kagome lattice}

Figure 1c shows the unit cell and translational vectors of the Kagome lattice; the periodic translational vectors are $\mathbf{a}_{1}=L[1, \sqrt{3}]$ and $\mathbf{a}_{1}=$ 


\begin{tabular}{|c|c|}
\hline edge & Internal force \\
\hline 1 & $\begin{array}{l}N=E_{s} A\left[\frac{A L^{2}+36 I_{z z}}{2 A L^{2}+24 I_{z z}} \epsilon_{x}+\frac{A L^{2}-12 I_{z z}}{2 A L^{2}+24 I_{z z}} \epsilon_{y}\right] \\
M=E_{s} I_{z z} \frac{6 A L-12 A x}{A L^{2}+12 I_{z z}} \gamma_{x y}\end{array}$ \\
\hline 2 & $\begin{array}{l}N=E_{s} A\left[\frac{A L^{2}}{2 A L^{2}+24 I_{z z}} \epsilon_{x}+\frac{A L^{2}+24 I_{z z}}{2 A L^{2}+24 I_{z z}} \epsilon_{y}+\frac{12 \sqrt{3} I_{z z}}{2 A L^{2}+24 I_{z z}} \gamma_{x y}\right] \\
M=E_{s} I_{z z}\left[\frac{6 \sqrt{3} A x-3 \sqrt{3} A L}{A L^{2}+12 I_{z z}} \epsilon_{x}+\frac{3 \sqrt{3} A L-6 \sqrt{3} A x}{A L^{2}+12 I_{z z}} \epsilon_{y}+\frac{6 A x-3 A L}{A L^{2}+12 I_{z z}} \gamma_{x y}\right]\end{array}$ \\
\hline 3 & $\begin{array}{l}N=E_{s} A\left[\frac{A L^{2}}{2 A L^{2}+24 I_{z z}} \epsilon_{x}+\frac{A L^{2}+24 I_{z z}}{2 A L^{2}+24 I_{z z}} \epsilon_{y}-\frac{12 \sqrt{3} I_{z z}}{2 A L^{2}+24 I_{z z}} \gamma_{x y}\right] \\
M=E_{s} I_{z z}\left[\frac{3 \sqrt{3} A L-6 \sqrt{3} x}{A L^{2}+12 I_{z z}} \epsilon_{x}+\frac{6 \sqrt{3} A x-3 \sqrt{3} A L}{A L^{2}+12 I_{z z}} \epsilon_{y}+\frac{6 A x-3 A L}{A L^{2}+12 I_{z z}} \gamma_{x y}\right]\end{array}$ \\
\hline
\end{tabular}

Table 6: Normal forces and bending moments of the hexagonal lattice

\begin{tabular}{|c|c|c|c|c|c|}
\hline \multicolumn{2}{|c|}{ model DoF } & \multicolumn{2}{c|}{ Normal Load err. } & \multicolumn{2}{c|}{ Shear err. } \\
\hline beam & plate & $u$ err & $v$ err & $u$ err & $v$ err \\
\hline 5418 & 800 & $6.25 \%$ & $1.83 \%$ & $1.81 \%$ & $2.02 \%$ \\
\hline 45018 & 800 & $1.40 \%$ & $0.26 \%$ & $1.15 \%$ & $1.43 \%$ \\
\hline
\end{tabular}

Table 7: Errors of plate models wrt lattice models, hexagonal lattice

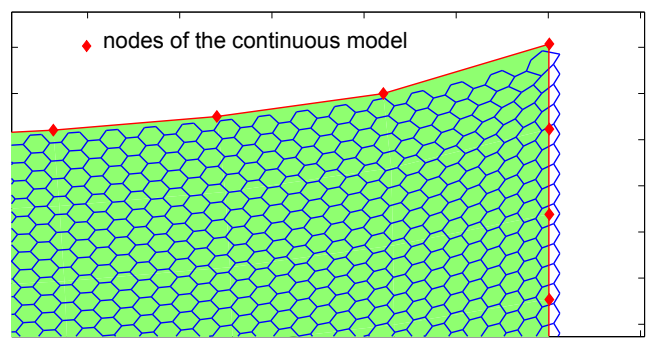

(a) Normal load, lattice and plate models

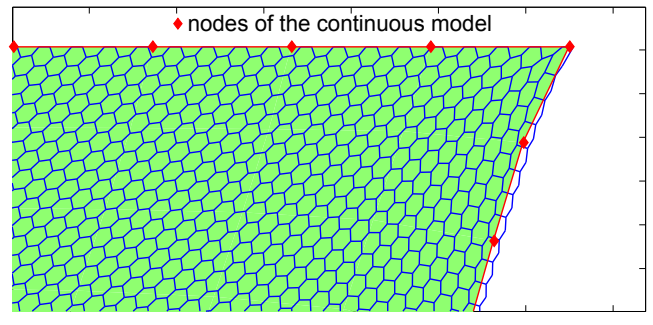

(b) Shear load, lattice and plate models

Figure 8: Deformed plate ("Detail" in Figure 4) made of hexagonal lattice with rigid joints

$L[1,-\sqrt{3}] ;$ the area of the uc is $S_{\mathrm{uc}}=2 \sqrt{3} L^{2} ;$ while $\mathbf{B}_{0}$ and $\mathbf{B}_{e}$ are given 
by equation 13. For the $\mathbf{B}_{e_{4}}$ and $\mathbf{B}_{e_{5}}$ sub-matrices it results

$$
\begin{aligned}
\mathbf{B}_{e_{4}}=\left[\begin{array}{ccc}
a_{2_{x}} & 0 & \frac{a_{2_{y}}}{2} \\
0 & a_{2 y} & \frac{a_{2 x}}{2} \\
0 & 0 & 0
\end{array}\right]=L\left[\begin{array}{ccc}
1 & 0 & \frac{-\sqrt{3}}{2} \\
0 & -\sqrt{3} & \frac{1}{2} \\
0 & 0 & 0
\end{array}\right] \\
\mathbf{B}_{e_{5}}=-\left[\begin{array}{ccc}
a_{1_{x}} & 0 & \frac{a_{1_{y}}}{2} \\
0 & a_{1_{y}} & \frac{a_{1 x}}{2} \\
0 & 0 & 0
\end{array}\right]=-L\left[\begin{array}{ccc}
1 & 0 & \frac{\sqrt{3}}{2} \\
0 & \sqrt{3} & \frac{1}{2} \\
0 & 0 & 0
\end{array}\right]
\end{aligned}
$$

Similarly to the hexagonal lattice, the following expression can be found for the $\mathbf{D}_{e}$ matrix

$$
\mathbf{D}_{e}^{T}=\left[\begin{array}{ccccccccccccccc}
\frac{L}{2} & 0 & 0 & 0 & 0 & \frac{\sqrt{3}}{4} & L & 0 & -\frac{\sqrt{3}}{4} & L & 0 & \frac{\sqrt{3}}{4} & 0 & 0 & -\frac{\sqrt{3}}{4} \\
0 & -\frac{\sqrt{3} L}{2} & 0 & 0 & 0 & -\frac{\sqrt{3}}{4} & 0 & 0 & \frac{\sqrt{3}}{4} & 0 & -\sqrt{3} L & -\frac{\sqrt{3}}{4} & 0 & -\sqrt{3} L & \frac{\sqrt{3}}{4} \\
-\frac{\sqrt{3} L}{4} & \frac{L}{4} & -\frac{1}{2} & 0 & 0 & \frac{1}{4} & 0 & \frac{L}{2} & \frac{1}{4} & -\frac{\sqrt{3} L}{2} & \frac{L}{2} & \frac{1}{4} & -\frac{\sqrt{3} L}{2} & 0 & \frac{1}{4}
\end{array}\right]
$$

The corresponding expression for the deformation work and the material stiffness matrix are

$$
\begin{gathered}
W=\frac{\sqrt{3} E_{s}}{16 L^{3}}\left[A\left(\gamma_{x y}{ }^{2}+3 \epsilon_{x}{ }^{2}+3 \epsilon_{y}{ }^{2}+2 \epsilon_{x} \epsilon_{y}\right) L^{2}+\right. \\
\left.I_{z z}\left(\gamma_{x y}{ }^{2}+\left(\epsilon_{x}-\epsilon_{y}\right)^{2}\right)\right] \\
\mathbf{K}=\frac{\sqrt{3} E_{s}}{8 L^{3}}\left[\begin{array}{ccc}
3\left(A L^{2}+2 I_{z z}\right) & A L^{2}-6 I_{z z} & 0 \\
A L^{2}-6 I_{z z} & 3\left(A L^{2}+2 I_{z z}\right) & 0 \\
0 & 0 & A L^{2}+6 I_{z z}
\end{array}\right]
\end{gathered}
$$

The above expression for the macroscopic stiffness of the Kagome lattice, in the hypothesis of pin jointed elements, $I_{z z}=0$, is in agreement with the results obtained by Hutchinson and Fleck (2006). With reference to the deformation work, the stretching and bending contributions are given by

$$
\begin{aligned}
& W_{s}=\frac{\sqrt{3} A E_{s}\left(\gamma_{x y}{ }^{2}+3 \epsilon_{x}{ }^{2}+3 \epsilon_{y}{ }^{2}+2 \epsilon_{x} \epsilon_{y}\right)}{16 L} \\
& W_{b}=\frac{3 \sqrt{3} E_{s} I_{z z}\left(\gamma_{x y}{ }^{2}+\left(\epsilon_{x}-\epsilon_{y}\right)^{2}\right)}{4 L^{3}}
\end{aligned}
$$

The eigenvalues of the stiffness matrix are still the same as those of the previous cases. Table 8 gives the expression of the $\frac{W_{b}}{W_{s}}$ ratios for the Kagome lattice.

Table 9 reports the internal forces for the Kagome lattice; due to the symmetries of the unit cell, the forces in the corresponding edges of the unit cell are equal. Figure 9 shows the lattice deformed shapes for the case of the Kagome rigid-jointed lattice. In Table 10 the differences between the beam and the plate models are reported; for the Kagome lattice, Figure 10 shows the deformed lattice under two different load conditions. 


\begin{tabular}{|l|c|c|c|}
\hline & $\boldsymbol{\epsilon}_{1}$ & $\boldsymbol{\epsilon}_{2}$ & $\boldsymbol{\epsilon}_{3}$ \\
\hline$\lambda$ & $\frac{\sqrt{3} E_{s} A}{2 L}$ & $\frac{\sqrt{3} E\left(A L^{2}+6 I_{z z}\right)}{4 L^{3}}$ & $\frac{\sqrt{3} E\left(A L^{2}+6 I_{z z}\right)}{16 L^{3}}$ \\
\hline$\frac{W_{b}}{W_{s}}$ & 0 & $\frac{6 I_{z z}}{A L^{2}}$ & $\frac{6 I_{z z}}{A L^{2}}$ \\
\hline
\end{tabular}

Table 8: Eigenvalues of the stiffness matrix and strain energy ratios of the Kagome lattice with rigid joints

\begin{tabular}{|c|c|}
\hline edges & Internal forces \\
\hline 1 and 4 & $\begin{array}{l}N=E_{s} A\left[\frac{1}{4} \epsilon_{x}+\frac{3}{4} \epsilon_{y}-\frac{\sqrt{3}}{4} \gamma_{x y}\right] \\
M=E_{s} I_{z z}\left[\left(\frac{\sqrt{3}}{L}-\frac{3 \sqrt{3}}{2 L^{2}} x\right) \epsilon_{x}+\left(\frac{3 \sqrt{3}}{2 L^{2}} x-\frac{\sqrt{3}}{L}\right) \epsilon_{y}+\frac{3}{2 L^{2}} \gamma_{x y} x\right]\end{array}$ \\
\hline 2 and 6 & $\begin{array}{l}N=E_{s} A \epsilon_{x} \\
M=E_{s} I_{z z}\left[-\frac{\sqrt{3}}{2 L} \epsilon_{x}+\frac{\sqrt{3}}{2 L} \epsilon_{y}+\left(\frac{3}{2 L}-\frac{3}{L^{2}} x\right) \gamma_{x y}\right]\end{array}$ \\
\hline 3 and 5 & $\begin{array}{l}N=E_{s} A\left[\frac{\epsilon_{x}}{4}+\frac{3 \epsilon_{y}}{4}+\frac{\sqrt{3} \gamma_{x y}}{4}\right] \\
M=E_{s} I_{z z}\left[\left(\frac{3 \sqrt{3}}{2 L^{2}} x-\frac{\sqrt{3}}{2 L}\right) \epsilon_{x}+\left(\frac{\sqrt{3}}{2 L}-\frac{3 \sqrt{3}}{2 L^{2}} x\right) \epsilon_{y}+\left(\frac{3}{2 L^{2}} x-\frac{3}{2 L}\right) \gamma_{x y}\right]\end{array}$ \\
\hline
\end{tabular}

Table 9: Normal forces and bending moments of the Kagome lattice

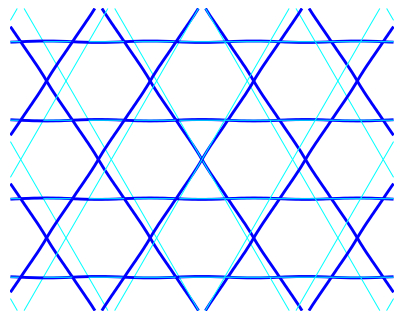

(a) $\epsilon_{x}>0, \epsilon_{y}=\gamma_{x y}=0$

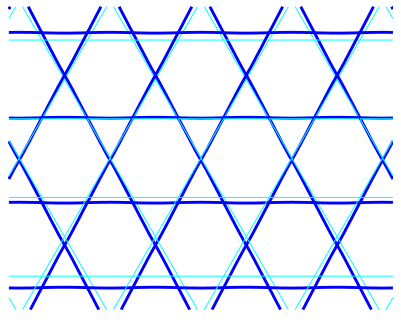

(b) $\epsilon_{y}>0, \epsilon_{x}=\gamma_{x y}=0$

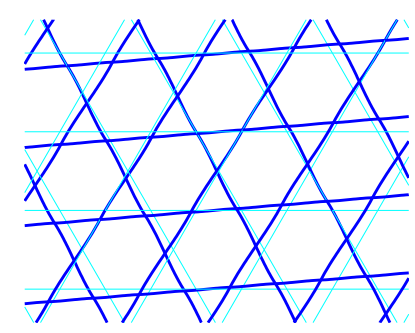

(c) $\gamma_{x y}>0, \epsilon_{x}=\epsilon_{y}=0$

Figure 9: Deformation modes of the Kagome lattice with rigid joints

\section{Conclusions}

A linear multiscale procedure for the analysis of lattice materials has been described and validated in this paper. The method allows the evaluation of the macroscopic stiffness of both pin-jointed and rigid-jointed lattices with 


\begin{tabular}{|c|c|c|c|c|c|}
\hline \multicolumn{2}{|c|}{ model DoF } & \multicolumn{2}{c|}{ Normal Load err. } & \multicolumn{2}{c|}{ Shear err. } \\
\hline beam & plate & $u$ err & $v$ err & $u$ err & $v$ err \\
\hline 6936 & 800 & $0.22 \%$ & $0.02 \%$ & $1.30 \%$ & $1.55 \%$ \\
\hline 27921 & 800 & $0.05 \%$ & $0.02 \%$ & $0.39 \%$ & $0.41 \%$ \\
\hline
\end{tabular}

Table 10: Errors of plate models wrt lattice models, Kagome lattice

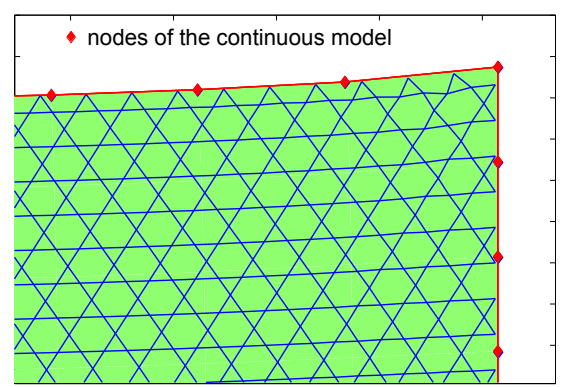

(a) Normal load, lattice and plate models

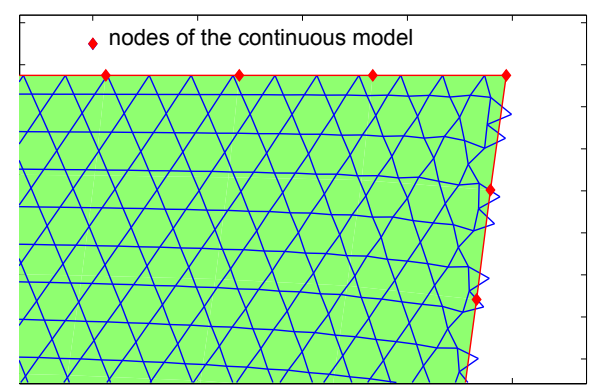

(b) Shear load, lattice and plate models

Figure 10: Deformed plate ("Detail" in Figure 4) made of Kagome lattice with rigid joints

arbitrary cell topology. The method permits also to determine the internal forces acting on each member of the lattice. The procedure focuses on the linear analysis of bidimensional lattices and it is illustrated with reference to three topologies: the triangular, the hexagonal and the Kagome. Further work is required to extend the analysis to tridimensional lattices with open and closed cell as well and to include the modelling of geometrical non linearity due to the lattice reorientation under an applied load.

For each topology, analytical expressions have been determined for the macroscopic in-plane stiffness constants and for the internal forces in the lattice edges. The results are in agreement with those found in the literature for the hexagonal and the Kagome lattice. To validate the procedure, the model of a rectangular portion of a discrete lattice has been compared with the model of a homogeneous rectangular domain of equivalent macroscopic stiffness, for prescribed dimensions, constraints and applied loads. The comparison of the displacements at the free boundaries has shown that the procedure described in this paper delivered a correct estimation of the stiffness of the lattice. 
The methodology presented in this paper allows readily express the lattice properties as a function of the cell parameters. Therefore, it can be easily integrated in an optimization framework for the optimum design of lattice materials.

\section{References}

Alderson, A., Alderson, K. L., 2007. Auxetic materials. Proceedings of the Institution of Mechanical Engineers Part G-Journal of Aerospace Engineering 221 (G4), 565-575.

Asaro, R. J., Lubarda, V. A., 2006. Mechanics of solids and materials. Cambridge University Press, Cambridge.

Ashby, M. F., 2005. Materials Selection in Mechanical Design, 3rd Edition. Elsevier.

Elsayed, M. S. A., Pasini, D., 2010a. Analysis of the elastostatic specific stiffness of $2 \mathrm{~d}$ stretching-dominated lattice materials. Mechanics of Materials $42(7), 709-725$.

Elsayed, M. S. A., Pasini, D., 2010b. Multiscale structural design of columns made of regular octet-truss lattice material. International Journal of Solids and Structures 47 (14-15), 1764-1774.

Gibson, L. J., Ashby, M. F., 1988. Cellular Solids: Structure and Properties.

Gibson, L. J., Ashby, M. F., Schajer, G. S., Robertson, C. I., 1982. The mechanics of two-dimensional cellular materials. Proceedings of the Royal Society of London Series a-Mathematical Physical and Engineering Sciences 382 (1782), 25-42.

Gonella, S., Ruzzene, M., 2008. Homogenization and equivalent in-plane properties of two-dimensional periodic lattices. International Journal of Solids and Structures 45 (10), 2897-2915.

Gonella, S., Ruzzene, M., 2010. Multicell homogenization of one-dimensional periodic structures. Journal of Vibration and Acoustics, Transactions of the ASME 132 (1), 0110031-01100311. 
Hutchinson, R. G., Fleck, N. A., 2006. The structural performance of the periodic truss. Journal of the Mechanics and Physics of Solids 54 (4), 756782 .

Kouznetsova, V., Geers, M. G. D., Brekelmans, W. A. M., 2002. Multiscale constitutive modelling of heterogeneous materials with a gradientenhanced computational homogenization scheme. International Journal for Numerical Methods in Engineering 54 (8), 1235-1260.

Kumar, R. S., McDowell, D. L., 2004. Generalized continuum modeling of 2-d periodic cellular solids. International Journal of Solids and Structures 41 (26), 7399-7422.

Lakes, R., 1987. Foam structures with a negative poisson's ratio. Science 235 (4792), 1038-1040.

Murr, L.E., G. S. M. F. L. H. M. E. M. B. H. D. M. L. L. M. W. R. B. J., 2010. Next-generation biomedical implants using additive manufacturing of complex cellular and functional mesh arrays. Philosophical Transactions of the Royal Society A: Mathematical, Physical and Engineering Sciences 368 (1917), 1999-2032.

Noor, A. K., 1988. Continuum modeling for repetitive lattice structures. Applied Mechanics Reviews 41 (7), 285-296.

Phani, A. S., Fleck, N. A., 2008. Elastic boundary layers in two-dimensional isotropic lattices. Journal of Applied Mechanics-Transactions of the Asme $75(2),-$.

Slaughter, W. S., 2002. The linearized theory of elasticity. Birkh user, Boston, Mass. [u.a.].

Spadoni, A., R. M., 2007. Static aeroelastic response of chiral-core airfoils. Journal of Intelligent Material Systems and Structures 18 (10), 1067-1075.

Stampfl, J., Fouad, H., Seidler, S., Liska, R., Schwager, F., Woesz, A., Fratzl, P., 2004. Fabrication and moulding of cellular materials by rapid prototyping. Int. Jour.. of Materials \& Product Technology 21 (4), 285-296.

Strang, G., 1988. Linear algebra and its applications, 3rd Edition. Harcourt Brace Jovanovich ; Brooks/Cole, Thomson Learning. 
Vigliotti, A., Pasini, D., 2011. Structural optimization of lattice materials. Accepted at the ASME 2011 International Design Engineering Technical Conferences \& Computers and Information in Engineering Conference, IDETC/CIE 2011, August 28-31, 2011, Washington, DC, USA.

Wang, A. J., Kumar, R. S., McDowell, D. L., 2005. Mechanical behavior of extruded prismatic cellular metals. Mechanics of Advanced Materials and Structures 12 (3), 185-200.

Wang, A. J., McDowell, D. L., 2004. In-plane stiffness and yield strength of periodic metal honeycombs. Journal of Engineering Materials and Technology-Transactions of the Asme 126 (2), 137-156.

Yang, S. F., Leong, K. F., Du, Z. H., Chua, C. K., 2002. The design of scaffolds for use in tissue engineering. part ii. rapid prototyping techniques. Tissue Engineering 8 (1), 1-11.

Zhu, H. X., Knott, J. F., Mills, N. J., 1997. Analysis of the elastic properties of open-cell foams with tetrakaidecahedral cells. Journal of the Mechanics and Physics of Solids 45 (3), 319-.

Zienkiewicz, O., Taylor, R. L., 2005. The Finite Element Method for Solid and Structural Mechanics, 6th Edition. Elsevier. 